\title{
Theoretical study of an evanescent optical integrated sensor for multipurpose detection of gases and liquids in the Mid-Infrared
}

\author{
Gutierrez-Arroyo Aldo 1, ", Baudet Emeline ${ }^{2}$, Bodiou Loic ${ }^{1}$, Nazabal Virginie ${ }^{2}$, Rinnert Emmanuel ${ }^{3}$, \\ Michel Karine ${ }^{4}$, Bureau Bruno ${ }^{2}$, Colas Florent ${ }^{3}$, Charrier Joel ${ }^{1}$
}

${ }^{1}$ FOTON, UMR CNRS 6082, ENSSAT, BP80518, F-22305 Lannion, France.

2 ISCR, UMR CNRS 6226, Glass \& Ceram Team, F-35042 Rennes, France.

3 IFREMER, Detect Sensors \& Measurements Lab, Technol Res \& Dev Dept, F-29280 Plouzane,

France.

${ }^{4}$ Bur Rech Geol \& Minieres, 3 Av C Guillemin, F-45060 Orleans 2, France.

*Corresponding author : Aldo Gutierrez-Arroyo, email address : mi.aldo.guti@gmail.com

\begin{abstract}
:
A theoretical study of evanescent optical sensor for multipurpose detection in the Mid-Infrared of gases and pollutants in water is presented in this paper. The opto-geometrical parameters of the transducers ridge waveguides - have been optimized in order to obtain the highest evanescent power factor for monomodal propagation in the Mid-Infrared. The highest sensitivity has been obtained for a configuration with an optimal length of waveguide Lopt $=4.3 \mathrm{~cm}$ for intrinsic propagation loss equal to $1 \mathrm{~dB} / \mathrm{cm}$. Then a spiral waveguide configuration is suggested to obtain this optical length path in a monolithic structure. A numerical example is also included using a ridge waveguide based on chalcogenide glasses (GeSbSe). In case of gas detection, a generic calculation of the minima concentrations to be detected as a function of the molar absorption for any working wavelength is presented. Extremely low limits of detection can be achieved due to the strong absorption coefficients of gases and chemical species in the Mid-Infrared spectral range, $268 \mathrm{ppb}$ in case of carbon dioxide at $\lambda=4.3 \mu \mathrm{m}, 1.848 \mathrm{ppm}$ and $781 \mathrm{ppb}$ for methane at $\lambda=3.31 \mu \mathrm{m}$ and at $\lambda=7.66 \mu \mathrm{m}$ respectively. For the pollutants detection in water, an improvement of the integrated structure has been proposed to avoid water absorption in this spectral region by deposing a polymer (PIB) as waveguide superstrate, thus the limit of detection for toluene is $26 \mathrm{ppb}$ at $\lambda=6.68 \mu \mathrm{m}$. These concentration minima that could be detected by the Mid-IR sensor are lower than the threshold limit values determined in the international environmental and health standards. Hence this integrated optical sensor may be considered as an attractive support tool in monitoring environmental and health fields.
\end{abstract}




\section{Highlights}

- Study of an evanescent optical integrated sensor in the Mid-IR. Optimization of the ridge waveguide design to obtain the highest sensitivity in the Mid-IR. A numerical example is included using a chalcogenide ridge waveguide. Limits of detections of ppb are achieved for liquids and gases in the Mid-IR.

Keywords : Mid-Infrared detection, Optical integrated sensor, Evanescent optical field, Gas detection, Liquid detection 


\section{Introduction}

The Mid-Infrared (MIR) is defined as the range of electromagnetic spectrum between $3 \mu \mathrm{m}$ and $20 \mu \mathrm{m}$. In the last years it has become a suitable solution for chemical sensing applications due to the excitation of fundamental vibrational transitions of several molecules in the gas phase or in the liquid phase [1]. Exploiting MIR has become a current topic for the industrial and research fields due to the wide variety of fingerprints of gases including $\mathrm{CO}_{2}, \mathrm{CO}, \mathrm{NO}_{2}, \mathrm{NO}, \mathrm{CH}_{4}$ and liquids such as acetone and emerging pollutants (BTEX Benzene, Toluene, Ethylbenzene, Xylene, HAP etc) [2-4], allowing quantitative, sensitive and selective detection.

The growing applications for optical sensors in the MIR have been based on novel low loss optical platforms. The laser sources emitting in this range of wavelengths are mainly the Quantum Cascade Laser (QCL). The mean light source providing a wide lasing wavelength beyond the near infrared $[5,6]$ and they represent one of the principal keys of development for spectroscopic applications in this spectral window. Moreover, the miniaturization of sensors has been achieved through the integrated optical devices combining high sensitivity and compactness, and a good degree of batch-production [7]. The optical integrated waveguides allow integration of optical and electrical functions on a single substrate, therefore lab on chip schemes have been proposed. Considering material optimization, these integrated systems have exhibited a strong transparence in 
the MIR and a high quality fabrication for various optical integrated structures. For instance, a silicon slot waveguide [8] with optical loss of $1.4 \mathrm{~dB} / \mathrm{cm}$ and silica ridge waveguides [9] with optical loss of $0.6 \mathrm{~dB} / \mathrm{cm}$ both functioning beyond $3 \mu \mathrm{m}$. Even when several platforms for the MIR have been probed in the recent years, such as germanium (Ge) [10] and gallium arsenide (GaAs) [11], the chalcogenide glasses (ChGs) present attractive properties. Owing to complete transparence in the whole MIR spectral window, capacity for rare earth doping and they are an excellent platform to manufacture photonic components because their feasibility to be deposed as thin films they emerge as ideal material candidate for sensing [12-14]. Recent works present integrated chalcogenide devices with MIR loss propagation, particularly ridge waveguides $[15,16]$ and high quality factor micro-cavities $[1,17,18]$. Furthermore, organic substances and antibodies have been detected using chalcogenide optical integrated devices, ethanol dissolved in cyclohexane [1] at $\lambda=5.2 \mu \mathrm{m}$ and antiSalmonella - anti-E. coli [19] using a FTIR in reflexion mode..

Accurate gas sensing is nowadays a crucial application for environmental and health care field. Spectroscopic based sensing techniques are the most employed, but they mean voluminous, complex and high costs setups, nevertheless integrated optical sensors are proposed as miniaturization, integration and low cost solution, beside an in situ and real time monitoring could be performed. In addition, optomicrofludic systems have been envisaged in order to encapsulate the testing gas to set as the superstrate waveguide. These configurations allow an efficient interaction with the evanescent optical field and it also furnishs of mechanical control of the gas [20]. Furthermore, quantity of organic pollutants in the aquatic environment, such as hydrocarbon compounds (aromatics, alkyl halides, phenols), has been increased in the last decades. Given the superlative importance of water and air resources for human being, this research topic is one of the highest challenges today facing scientists. Accordingly, there is a growing concern to develop detection systems to favor miniaturized and in situ devices based on different technologies than usual sensors exploiting attenuated total reflectance (ATR). Despite the enormous potential of detection of several water pollutant substances in the MIR, the development of sensors has been limited by the strong absorption of water in this spectral region, 
which causes a high attenuation in the transmitted optical signal. This fact represents a serious drawback to detect $\mathrm{ppb}-\mathrm{ppm}$ concentrations of pollutants, which are the typical concentrations ranges in water samples and different strategies have been employed like using a layer of polymer associated with a detection by evanescent field [21].

In this paper, an analytical design of an evanescent optical field sensor composed by a ridge waveguide working in the MIR spectral domain is provided. This model can provide a general platform for multipurpose sensing, being suitable to detect gas substances, as well as dissolved substances in aquatics media. The objective is to present a generic calculation for all substances (molar absorptions $\varepsilon$ ) and wavelengths $\lambda(\mu \mathrm{m})$, nevertheless it will focus on cases where strong absorption peaks are present for certain gas and liquid substances at a single fixed wavelength. Low limits detection of concentration of gas and emerging water pollutants are aimed to be achieved with the configuration proposed, particularly for methane and carbon dioxide in gas applications and for toluene dissolved in water in the liquid medium case. A lower limit of detection than the threshold limit value (TLV) determined by the international environmental standards is expected: $0.7 \mathrm{ppm}, 1000 \mathrm{ppm}, 1000 \mathrm{ppm}$ for toluene [22], carbon dioxide [23,24] and methane [25,26] respectively. Thus, an optimization of the ridge waveguide optical and geometrical parameters will be performed to set the best configuration in terms of evanescent field interaction for monomodal propagation. The objective is to present a generic calculation for all substances (molar absorptions $\varepsilon$ ) and wavelengths $\lambda$ $(\mu \mathrm{m})$, nevertheless it will focus on cases where strong absorption peaks are present for certain gas and liquid substances at a single fixed wavelength.

\section{Theoretical Background: Evanescent optical detection in gas and liquid media}

A commonly exploited operating principle in photonic chemical sensor is the optical absorption. The absorbance $A$ is a physical parameter which is a function of the concentration of substance $C\left(\mathrm{~mol} \mathrm{~L}^{-1}\right)$ and the molar absorption $\varepsilon\left(\mathrm{mol}^{-1} \mathrm{~L} \mathrm{~cm}^{-1}\right): A=C \varepsilon\left(\mathrm{cm}^{-1}\right)$. In optical integrated sensors, this sensing principle is useful to associate the optical signal power $P$ and the solute concentration $C$ through the Beer-Lambert law [27]: 
$P=P_{0} \exp \left(-\eta \varepsilon C L-\alpha_{\text {prop }} L\right)$

Where $P_{0}(\mathrm{~W})$ is the optical power at the waveguide input, $\alpha_{\text {prop }}\left(\mathrm{cm}^{-1}\right)$ is the waveguide intrinsic propagation optical loss, $L(\mathrm{~cm})$ is the waveguide length (optical path) and $\eta(\%)$ is the evanescent power factor. Sensing mechanism consists in monitoring the output optical signal power, which changes as a function of the concentration $C\left(\mathrm{~mol} \mathrm{~L}^{-1}\right)$. Consequently, the power attenuation increases with higher levels of the solute concentration, but also it is directly dependent of $\eta$.

The evanescent power is the part of optical field non-confined in the waveguide guiding layer and it determines the interaction degree with the superstrate media [28]. It is expressed by the evanescent power factor $\eta$ and it is given by the expression:

$\eta=\frac{P_{\text {sup }}}{P_{\text {total }}}$

Where $\mathrm{P}_{\text {sup }}$ and $\mathrm{P}_{\text {total }}$ are the Poynting vectors (representing the energy flux of the propagated electromagnetic field in the superstrate) and the guided light total power respectively [29].

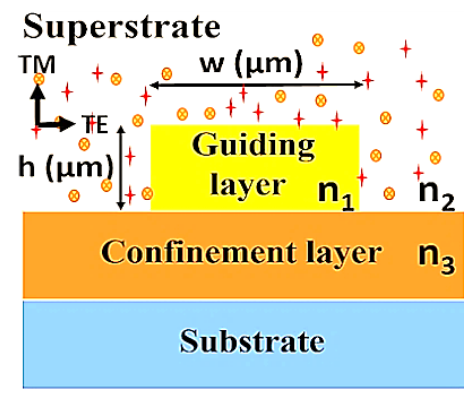

Fig. 1. Schematic of a ridge waveguide and the interaction with the molecules in the superstrate.

The penetration depth $d_{\mathrm{p}}$ is the interaction distance from the $\mathrm{n}_{1}-\mathrm{n}_{2}$ interface where the intensity of the evanescent optical field decreases at 1/e ratio into the superstrate medium [30]. Hence, the penetration depth $d_{\mathrm{p}}$ is proportional to $\eta$, and it may be defined as a function of the wavelength of incident light $\lambda_{0}$, the refraction index of the superstrate $n_{2}$ and guiding layer $n_{1}$ (Fig. 1) and the angle of incidence $\theta$, it is described in Eq (3). 


$$
d_{p}=\frac{\lambda_{0}}{2 \pi \sqrt{n_{1}^{2} \sin ^{2} \theta-n_{2}^{2}}}
$$

Thereafter, the sensitivity of the device is represented by the variation of output optical power as a function of the solute concentration $C$, it could be calculated differentiating Eq. (1) with respect to $C$ [29]:

$S=\left|\frac{d P}{d C}\right|=\varepsilon \eta L P_{0} \exp \left(-\varepsilon \eta C L-\alpha_{\text {prop }} L\right)$

Sensitivity is also useful to determine the optimum waveguide length $\mathrm{L}_{\text {opt }}$, which is found at the maximum of the $S$ function, then $\mathrm{L}_{\text {opt }}$ can be calculated as follows:

$$
L_{o p t}=\frac{1}{\eta \varepsilon C+\alpha_{p r o p}}
$$

The minimum solute concentration $C_{\min }$ that could be measured by the sensor represents the limit of detection (LOD). The goal of this theoretical study is to design of a device for which the LOD is lower than the threshold limit value (TLV) determined by the international environmental standards. Therefore, the minimum optical power $P_{\min }$ that could be detected by the sensor is modeled as the difference between the optical power for a null concentration $\mathrm{P}(C=0)$ and the optical power for the limit of detection $\mathrm{P}\left(C_{\min }\right)$ :

$$
P_{\min }=P(C=0)-P\left(C_{\min }\right)
$$

The LOD is physically limited by the performance characteristics of the photodetector, then certains parameters given by the employed photodetector in the experimental setup are consider to calculate the minimum solute concentration $C_{\min }$, such as the Noise Equivalent Power (NEP), the bandwidth (B) and arbitrary Signal to Noise Ratio (SNR) which takes into consideration the coupling losses at the waveguide input and output. Thus, using the Lambert-Beer law (Eq. 1), it is possible to develop the Eq. (6) and $C_{\min }$ could be expressed as follows:

$$
C_{\min }=\frac{-\ln \left[1-\frac{S N R N E P \sqrt{B}}{P_{0} \exp \left(-\alpha_{\text {prop }} L_{\text {opt }}\right) \exp \left(-\eta \varepsilon_{\text {Solvent }} C_{\text {Solvent }} L_{\text {opt }}\right)}\right]}{\varepsilon_{\text {analyte }} \eta L_{\text {opt }}}
$$


In a particular case, the solvent medium may be optically non-absorbing at the working wavelength, then a simplification in Eq. (7) leads to the following expression:

$C_{\min }{ }^{\prime}=\frac{-\ln \left[1-\frac{\operatorname{SNR} N E P \sqrt{B}}{P_{0} \exp \left(-\alpha_{p r o p} L_{o p t}\right)}\right]}{\varepsilon \eta L_{o p t}}$

\section{Simulation Results and Discussion}

Several organic and inorganic substances such as gases and liquids are characterized by their strong absorption in the MIR, in this work the detection of methane $\left(\mathrm{CH}_{4}\right)$ at $\lambda=3.31 \mu \mathrm{m}$ and $\lambda=7.66 \mu \mathrm{m}$, and carbon dioxide $\left(\mathrm{CO}_{2}\right)$ at $\lambda=4.3 \mu \mathrm{m}$ for sensing gases are analyzed. Furthermore, a study of detection in liquid medium will be presented, in particular toluene dissolved into water at $\lambda=6.68 \mu \mathrm{m}$, but an analysis taking into account high absorption of water must be firstly made in order to determine the feasibility of pollutants detection.

For the design of the MIR evanescent optical sensor, computer simulations based on the effective index method were firstly performed in order to determine the geometrical dimensions for a single mode ridge waveguide. Obtaining the highest evanescent power factor $\eta$ and propagating simultaneously in monomodal regime in the MIR was aimed. The structure of the integrated platform was composed by a guiding layer for which the refraction index is $n_{1}=2.81$ at $\lambda=4-8 \mu \mathrm{m}$ and a confinement layer with a refraction index of $n_{3}=2.40$ at $\lambda=4-8 \mu \mathrm{m}$, both layers were selected from different compositions of $\left(\mathrm{GeSe}_{2}\right)_{100-x}\left(\mathrm{Sb}_{2} \mathrm{Se}_{3}\right)_{x}$ chalcogenide glasses [31]. A thickness of $5 \mu \mathrm{m}$ for the confinement layer was taken into consideration to avoid radiation losses through the substrate. The superstrate is totally dependent of the substance to be detected, then the air will be assumed as the superstrate for the gas sensor and the water for case of liquids detection. The refractive index of the air [32] is $n_{2}=1.0002$ at $\lambda=4-8 \mu \mathrm{m}$, for water [33] are 1.45 at $\lambda=3.31 \mu \mathrm{m}$ and 1.329 at $\lambda=6.68 \mu \mathrm{m}$. 
The evolution of $\eta$ as a function of the geometrical parameters (width and height) of chalcogenide waveguide which allow a single mode propagation is presented below in Fig. 2(a), (b) at $\lambda=4.3 \mu \mathrm{m}$ and $\lambda=7.66 \mu \mathrm{m}$ wavelengths of interest for $\mathrm{CO}_{2}$ and $\mathrm{CH}_{4}$, respectively. The Fig. 2(c) shows the case of water as superstrate at $\lambda=6.68 \mu \mathrm{m} . \mathrm{TM}$ polarization was considered for all these calculations.
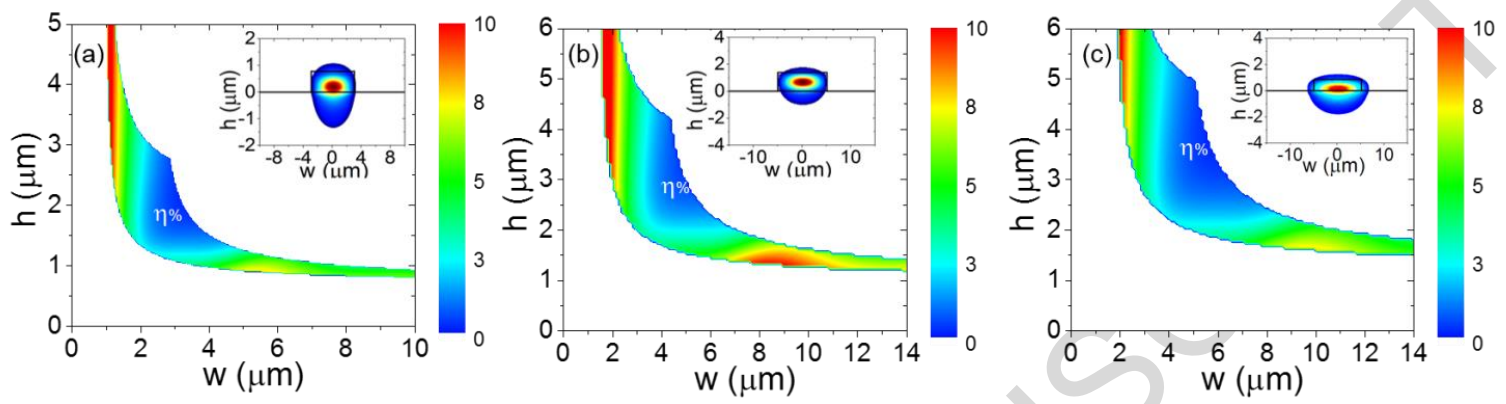

Fig. 2. (a) Evolution of the evanescent power factor $\eta$ as a function of $w, h$ for monomodal propagation in the detection of any gas at $\lambda=4.3 \mu \mathrm{m}$, (b) at $\lambda=7.66 \mu \mathrm{m}$ and (c) for any substance dissolved in water at $\lambda=6.68 \mu \mathrm{m}$. The fundamental mode TM00 intensity profile for the optima geometrical parameters (width $w$ and height $h$ ) of chalcogenide waveguide is included for every case.

TM polarization represents an optimization in the sensor design by maximizing the evanescent power factor. In this case, the vertical component of the electrical field enhances the interaction between the propagated mode and the superstrate medium in the top of the guiding layer. TE polarization assumes a horizontal electrical field which leads to a low interaction between the horizontal evanescent field of guided mode and the superstrate due to the guiding layer widths are larger than the heights. Furthermore, the best configurations were always found for a low thickness and high widths according to the fabrication feasibility. In this context, the width and height for a monomodal ridge waveguide have been determined from the highest $\eta$. These values are shown in the Table 1 . The penetration depths $d_{\mathrm{p}}$ have been also reported in Table 1 , and it can be seen that the penetration depth increases as the wavelength increases. 
Table 1. Penetration distance, effective index and evanescent power factor $\eta$ as a function of the optimum geometrical parameters of ridge waveguide $(w, h)$ for monomodal propagation in the detection of methane $\left(\mathrm{CH}_{4}\right)$, carbon dioxide $\left(\mathrm{CO}_{2}\right)$ and toluene dissolved in water.

\begin{tabular}{ccccccc}
\hline Molecule to be detected & $\begin{array}{c}\text { Wavelength } \\
(\mu \mathrm{m})\end{array}$ & $\begin{array}{c}W \\
(\mu \mathrm{m})\end{array}$ & $\begin{array}{c}h \\
(\mu \mathrm{m})\end{array}$ & $\begin{array}{c}\eta \\
(\%)\end{array}$ & $\begin{array}{c}n_{\text {eff } T M} \\
(\mathrm{~nm})\end{array}$ \\
\hline $\mathrm{CO}_{2}$ & 4.30 & 6 & 0.8 & 8 & 2.4046 & 313 \\
$\mathrm{CH}_{4}$ & 3.31 & 5 & 0.6 & 8 & 2.4050 & 240 \\
$\mathrm{CH}_{4}$ & 7.66 & 10 & 1.7 & 8 & 2.4338 & 550 \\
Toluene & 6.68 & 9 & 1.4 & 9 & 2.4223 & 547 \\
\hline
\end{tabular}

The evanescent power factor is determined by the refractive index contrast of the media (superstrate, guiding layer and confinement layer). Then, modifying the refractive index of chalcogenide glasses implies a variation of the interaction ratio between the optical non-confined field in the guiding layer and the superstrate medium, hence the sensitivity (Eq. 4) and the LOD (Eq. 8) will be directly affected.

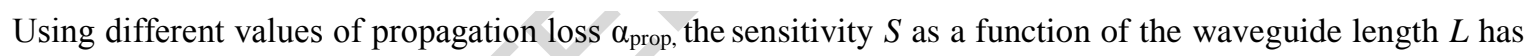
been plotted in Fig. 3(a) using Eq. (4). The calculation considers $\eta=8 \%, \mathrm{P}_{0}=1 \mathrm{~mW}$ (a realistic waveguide output power) and values of $\mathrm{C}, \varepsilon$ which fulfil the next condition: the product of evanescent power factor, molar absorption coefficient and concentration of solute is always lower than the optical propagation loss value. It is noteworthy that the design of sensor is focus in low concentration (order of ppm) and realistic values of molar absorption are comprises between $\varepsilon=10^{1}-10^{4} \mathrm{~mol}^{-1} \mathrm{~L} \mathrm{~cm}^{-1}$. 

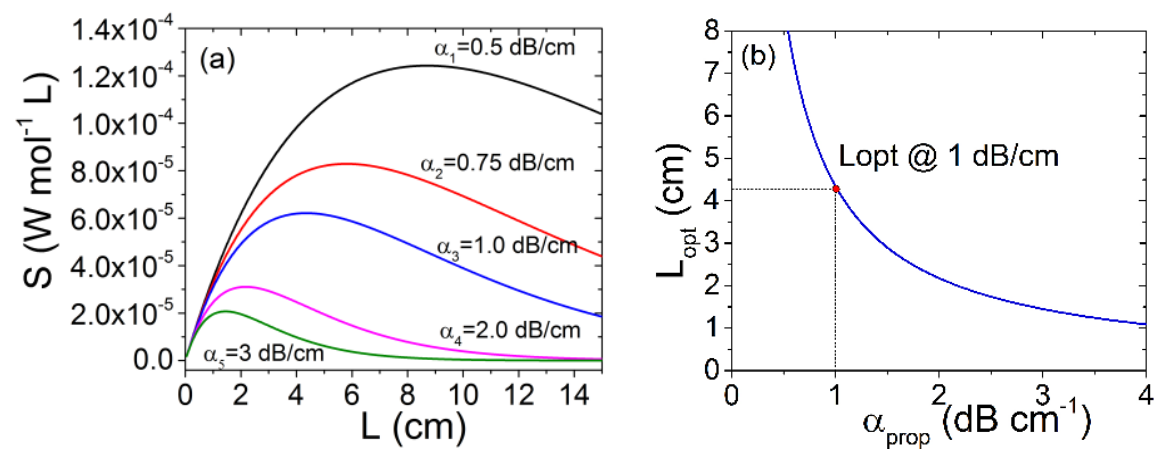

Fig. 3. (a) Sensitivity of the integrated sensor as a function of the waveguide length for several values of propagation loss in the detection of low concentrations gas and liquids in the MIR; (b) Optimal waveguide length as function of propagation loss for the detection of low concentration at $\lambda=\mathrm{MIR} \mu \mathrm{m}$.

The importance of loss propagation of waveguide in the performance of the sensor is shown in the curves of sensitivity (Fig. 3(a)). Lower propagation losses allow a better interaction between the propagated light and the molecules in the superstrate and lead to a higher sensitivity. The propagation losses are decisive to determine the optimum waveguide length $\mathrm{L}_{\text {opt }}$, this dimension is longer when $\alpha_{\text {prop }}$ are weaker . From the evolution of the optimal length $\mathrm{L}_{\text {opt }}$ as a function of the propagation loss $\alpha_{\text {prop, }}$ (Fig. 3(b)), it is possible to set $\mathrm{L}_{\text {opt }}=4.3 \mathrm{~cm}$ for $\alpha_{\text {prop }}=1 \mathrm{~dB} / \mathrm{cm}$, which is a realistic but unfavorable propagation loss value of chalcogenide glasses in the MIR. In order to reached a waveguide length about $4 \mathrm{~cm}$, this length could be obtained by the fabrication of a spiral waveguide of small dimension of small dimension [34]. Finally, determining the optimal length $\mathrm{L}_{\text {opt }}$ of ridge waveguide allows to define the dimensions of the transducer in chalcogenide to be fabricated.

\subsection{Gas detection}

In this paper, the feasibility of sensing gas substances for very low detection limits will be demonstrated thanks to strong absorption lines in the MIR. Thus, the detection of methane $\left(\mathrm{CH}_{4}\right)$ at $\lambda=3.31 \mu \mathrm{m}$ and $\lambda=7.66$ $\mu \mathrm{m}$, and carbon dioxide $\left(\mathrm{CO}_{2}\right)$ at $\lambda=4.3 \mu \mathrm{m}$ (Fig. 4(a)) will be explored. 

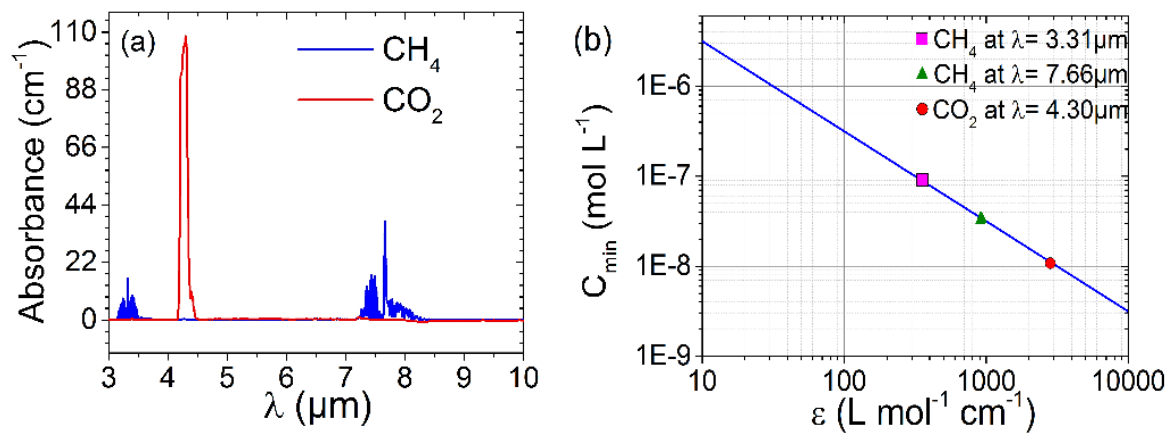

FIG. 4. (a) MIR absorbance spectra of (a) methane [35] - $\mathrm{CH}_{4}-$, carbon dioxide [28] - $\mathrm{CO}_{2}$ - under normal conditions of pressure and temperature ( $1 \mathrm{~atm}, \mathrm{~T}=273 \mathrm{~K}$ ). (b) Minimum solute concentration of any gas detected in the MIR as a function of the molar absorption coefficient detected by the evanescent optical sensor.

A generic calculation of the minimum solute concentration $C_{\min }$ ' (Eq. (8)) as a function of the molar absorption coefficient $\varepsilon$ is presented for any absorbing gas in the MIR detected by the evanescent optical sensor (Fig. 4(b)). The results are valid for gas detection at any working wavelength. Then, in order to estimate the limit of gas detection (limit of gas detection LOD) in a real experimental case in a real case, a photodetector has been chosen, it is the DSS-MCT14 020L from Horiba for which size is $2 \times 2 \mathrm{~mm}^{2}$ and cooled by liquid nitrogen. The operation spectral range of this MCT photodetector is from 3 to $12 \mu \mathrm{m}$, the noise equivalent power is $N E P=5 \times 10^{-12} \mathrm{~W} \mathrm{~Hz}^{-1 / 2}$, the bandwidth is $B=5 \mathrm{KHz}$. The Signal to Noise Ratio (SNR) is fixed equal to 10 for this calculation. This SNR value is unfavorable considering the coupling loss at the waveguide input and output. An evanescent power factor previously calculated for gases $\eta=8 \%$ (from Fig. 2(b), 2(c), a typical QCL's output power of $1 \mathrm{~mW}\left(\mathrm{P}_{0}\right)$, propagation loss of chalcogenide glasses in the MIR of $1 \mathrm{~dB} / \mathrm{cm}\left(\alpha_{\mathrm{prop}}\right)$ and the optimum waveguide length $L_{\mathrm{opt}}=4.3 \mathrm{~cm}$ are considered. The results of gas minimum concentration $\left(\mathrm{C}_{\min }\right)$ are presented in Table 2 . 
Table 2. Limit of detection of the evanescent optical field sensor given by the minimum detectable concentration $C_{\min }$ of carbon dioxide and methane in the MIR.

\begin{tabular}{|c|c|c|c|c|c|}
\hline & Wavelength & $\varepsilon$ & $C_{\min }$ & $C_{\min }$ & $T L V^{\mathrm{a}}$ \\
\hline Molecule to be detected & $(\mu m)$ & $\left(\mathrm{L} \mathrm{mol}^{-1} \mathrm{~cm}^{-1}\right)$ & $\left(\mathrm{mol} \mathrm{L}^{-1}\right)$ & (ppm) & $(\mathrm{ppm})$ \\
\hline $\mathrm{CO}_{2}$ & 4.30 & 2613 & $1.2 \times 10^{-8}$ & 0.268 & 1000 \\
\hline $\mathrm{CH}_{4}$ & 3.31 & 382.5 & $8.25 \times 10^{-8}$ & 1.848 & 1000 \\
\hline $\mathrm{CH}_{4}$ & 7.66 & 904.22 & $3.489 \times 10^{-8}$ & 0.781 & 100 \\
\hline
\end{tabular}

Hence, the higher absorption of the substance in the superstrate $(\varepsilon)$, the lower minimum concentration $\mathrm{C}_{\min }$ is. The LOD for carbon dioxide and methane (in ppm) are 3 orders of magnitude lower than the Threshold Limit Value and then the LOD estimated for the Siebert's configuration ${ }^{28}$ respectively. Then, the designed sensor exhibits a remarkable performance in gas sensing and it could be implemented for any other absorbing gas in the MIR. These results probe the great potential in the environmental monitoring field and they suggest that the evanescent optical field sensor could be proposed as a threshold alarm tool.

\subsection{Detection of substances in aquatic environment}

To illustrate the detection of pollutant agents into water, o illustrate the detection of pollutant agents into water, the toluene which is an aromatic hydrocarbon with solubility in water of $535 \mathrm{ppm}$ [22], was selected for this purpose was selected for this purpose. The toluene is used in the chemical industry as a solvent especially for paints, oils and resins, and as a raw material in the production of benzene, phenol and other organics substances. Long exposures to this substance could be related to different diseases of nervous system, kidneys, liver and it has been identified as a carcinogenic agent $[36,37]$. The World Health Organization has determined $0.7 \mathrm{ppm}$ of toluene in drinking water as the maximum contaminant level.

In order to guarantee the feasibility of detection of pollutants dissolved in water, an analysis of optical attenuation caused by the water absorption in the MIR as a function of the waveguide length is required (Fig. 5(a)). Although the method is perfectly generalizable, this present study focuses, for instance, on the absorption peak of toluene at $\lambda=6.68 \mu \mathrm{m}$ presented in Fig. 5(b). For this calculation, Eq. (1) is used 
considering the following set of realistic parameters: an evanescent power factor $\eta=9 \%$ (from Fig. 2 (c)), an intrinsic propagation loss $\alpha_{\text {prop }}=1 \mathrm{~dB} / \mathrm{cm}$, a concentration of water $\mathrm{C}_{\mathrm{water}}=5.50 \mathrm{~mol} \cdot \mathrm{L}^{-1}=100 \mathrm{v} / \mathrm{v} \%$, a molar absorption coefficient of water $\varepsilon_{\text {water }}=11.38 \mathrm{~mol}^{-1} \mathrm{~L} \mathrm{~cm}^{-1}$ at $\lambda=6.68 \mu \mathrm{m}$. Once more, the performance characteristics of the photodetector must be considered to set the lowest optical signal (LOS) to be detected, which is given as follows: $\mathrm{LOS}=\mathrm{SNR} * \mathrm{NEP} * \mathrm{~B}^{1 / 2}=3.5 \mathrm{nW} . \mathrm{L}_{\max }=2.5 \mathrm{~mm}$ is obtained as the waveguide length which causes an attenuation of output power equivalent of LOS, hence for detection of pollutant substances in aquatic environments a ridge waveguide shorter than $\mathrm{L}_{\max }$ must be fabricated. Moreover, from Eq. (5) the optimum waveguide length for detection in aquatic media is estimated to be: $\mathrm{L}_{\mathrm{opt} \_\mathrm{aq}}=176 \mu \mathrm{m}$.
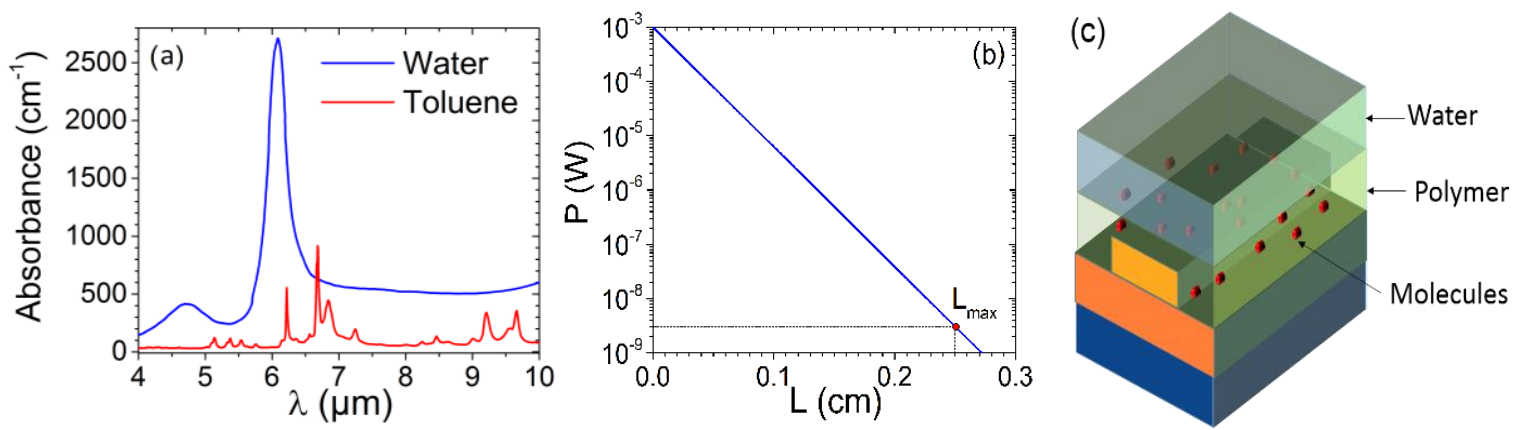

FIG. 5. (a) MIR absorbance spectra of Toluene [38] at $100 \% \mathrm{v} / \mathrm{v}$ and water [39] at $\mathrm{T}=278 \mathrm{~K}$. (b) Power transmitted response as a function of the waveguide length taking into consideration the strong absorbance of water in the MIR. (c) Schematic of waveguide functionalization: an optical non-absorbing in the MIR polymer is deposed as superstrate for detection of dissolved pollutants into water.

The minimum concentration of toluene dissolved in water at $\lambda=6.68$ is calculated from Eq. (7) and $\mathrm{C}_{\text {min_toluene }}=6.20 \times 10^{-5} \mathrm{~mol} \mathrm{~L}^{-1}=5.7 \mathrm{ppm}$ is obtained. This calculation considers a molar absorption of toluene equal to $\varepsilon_{\text {toluene }}=97.4 \mathrm{~mol}^{-1} \mathrm{~L} \mathrm{~cm}^{-1}$. A high concentration of water is considered $\mathrm{C}_{\text {water }}=55.4945 \mathrm{~mol} \mathrm{~L}^{-1}=99.99$ $\mathrm{v} / \mathrm{v} \%$, the complementary parameters such as $\mathrm{L}_{\mathrm{opt} \_\mathrm{q}}=176 \mu \mathrm{m}$. and $P_{0}, \eta, \alpha_{\mathrm{prop}}, S N R, N E P, B$ are the same values from the previous calculation. Even when $\mathrm{C}_{\min }$ toluene is lower than the solubility threshold value (535 ppm), this result is not enough to consider this sensor as a threshold alarm tool because its LOD is higher than the maximum contaminant level in drinking water. 
Therefore a second design for a sensor detecting in aquatic environments is presented, the LOD could be enhanced by functionalizing the superstrate of waveguide. The concept restricts the water absorption in the MIR by replacing water in contact with the surface of the chalcogenide waveguide by an optical nonabsorbing material, particularly by a polymer like the Polyisobutylene (PIB) [40], which is an hydrophobic polymer with a high transmittance in this range of wavelength. The optimized waveguide structure takes advantage of permeability of the polymer to extract the analyte molecules from the solution and to enable them to diffuse to be in contact to the guiding layer, then it is possible to detect them by optical evanescent field absorption [41], as it is shown in the Fig. 5(c). The structure of the hydrophobic polymer (PIB) is not strongly modified after exposing to analyte, thus the detectivity remains in the same order of magnitude. Moreover, the diffusion into the PIB leads to an accumulation effect of the solute molecules in vicinity of the evanescent field increasing the sensor performance [42].

Thus, the expected minimum detectable concentration of toluene dissolved in water detected by using the polymer functionalization is $\mathrm{C}_{\text {min_toluene } \mathrm{PIB}}=2.8345 \times 10^{-7} \mathrm{~mol} \mathrm{~L}^{-1}=26 \mathrm{ppb}$, it was calculated from the Eq. (8) assuming only the absorption coefficients of toluene at $\lambda=6.68 \mu \mathrm{m}$, the coefficients $P_{0}, \eta, \alpha_{\text {prop }}, S N R, N E P, B$ previously applied and the optimal length of waveguide for an optical non-absorbing medium $\mathrm{L}_{\text {opt }}=4.3 \mathrm{~cm}$ (Fig. 3(b)). This optimization in the sensor design allows enhancing the LOD from ppm to ppb range. This result is noticeably lower than the maximum level of concentrations suggested by the international standards of quality of water and the MIR sensor could be furnished as an efficient monitoring tool.

\section{Conclusion}

A generic model of an evanescent optical field sensor able to detect gases and substances dissolved in water in the MIR have been introduced. Ridge waveguides are proposed to be the transducers, and their optima dimensions have been determined using the Effective Index Method in order to provide the highest evanescent field factor for monomodal propagation. From the sensitivity of the device, it has been possible to determine the optimal length waveguide for any value of intrinsic loss propagation. A numerical example has 
been given using a ridge waveguide base on (Ge-Sb-Se) chalcogenide glasses in gas detection for carbon dioxide at $\lambda=4.3 \mu \mathrm{m}$, methane at $\lambda=3.31 \mu \mathrm{m}$ and at $\lambda=7.66 \mu \mathrm{m}$, and the limits of detection have been calculated as $268 \mathrm{ppb}, 1.848 \mathrm{ppm}$ and $781 \mathrm{ppb}$ respectively. For these applications the optimal length waveguide is $L_{\mathrm{opt}}=4.3 \mathrm{~cm}$ for $\alpha_{\mathrm{prop}}=1 \mathrm{~dB} / \mathrm{cm}$, then a spiral waveguide configuration could be fabricated to obtain this optical length path in an integrated sensor. In addition, a general calculation of minima gas concentrations as function of the molar absorption is presented, it considers wavelength non-dependence.

For detection of substances dissolved in liquid media, an optimization in the integrated structure suggests to deposit a functionalized hydrophobic polymer as a waveguide superstrate to avoid water absorption in the MIR. Consequently, the LOD is enhanced is enhanced for the detection of dissolved substances in water, for example, with this configuration $26 \mathrm{ppb}$ of toluene can be can be achieved to be measured at $\lambda=6.68 \mu \mathrm{m}$.

All these numerical results allow to present this optical sensor as an alarm tool in the monitoring processes because the resolution could operate under the threshold limit value determined in the international environmental standards and they established the promising first step towards the development of integrated optical sensor working in the MIR.

\section{Acknowledgements}

CONACyT (Mexican Council for Science and Technology) is gratefully acknowledged for sponsoring the grant of the author.

\section{References}

[1] V. Singh, P. T. Lin, N. Patel, H. Lin, L. Li, Y. Zou, F. Deng, C. Ni, J. Hu, J. Giammarco, P. Soliani, B. Zdyrko, I. Luzinov, S. Novak, J. Novak, P. Wachtel, S. Danto, J. D. Musgraves, K. Richardson, L. C. Kimerling, A. M Agarwal. Sci. Technol. Adv. Mater. 15 (2014) 014603.

[2] F. K. Tittel, D. Richter, A. Fried. Topics Appl. Phys. 89 (2003) 445-516.

[3] R. Silverstein, F. Webster, D. Kiemle. Spectrometric Identification of Organic Compounds. Willey (2005) 88-108. 
[4] M.B. Sajid, T. Javed, A. Farooq. J. Quant. Spectrosc. Radiat. Transfer. 155 (2015) 66-74.

[5] B. Mizaikoff. Chem. Soc. Rev. 42 (2013) 8683-8699.

[6] B. G. Lee, M. A. Belkin, R. Audet, J. MacArthur, L. Diehl, C. Pflügl, F. Capasso, D. C. Oakley, D. Chapman, A. Napoleone, D. Bour, S. Corzine, G. Höfler, J. Faist. Appl. Phys. Lett. 91 (2007) 231101.

[7] L. M. Lechuga. Quimica Analitica. 19 (2000) 61-67.

[8] J. Soler-Penadés, A. Z. Khokhar, M. Nedeljkovic, G. Z. Mashanovich. IEEE Photon. Technol. Lett. 27 (2015) 11.

[9] G. Mashanovich, M. M. Milošević, M. Nedeljkovic, N. Owens, B. Xiong, E. J. Teo, Y. Hu. Opt. Express. $19(2011) 8$

[10] R. Soref. Nat. Photonics 4 (2010) 495 - 497.

[11] M. Sieger, F. Ballu, X. Wang, S. Kim, L. Leidner, G. Gauglitz, B. Mizaikoff. Anal. Chem. 85 (2013) 3050-3052.

[12] B. Eggleton. Opt. Express 18 (2010) 25.

[13] K. Richardson, D. Krol, K. Hirao. International Journal of Applied Glass Science 1 (2010) 74-86.

[14] V. Nazabal, M. Cathelinaud, W. Shen, P. Nemec, F. Charpentier, H. Lhermite, M. L. Anne, J. Capoulade, F. Grasset, A. Moreac, S. Inoue, M. Frumar, J. L. Adam, M. Lequime, C. Amra. Appl. Opt. 47 (2008) 13.

[15] P. Ma, D. Y. Choi, Y. Yu, X. Gai, Z. Yang, S. Debbarma, S. Madden, B. Luther-Davies. Opt. Express. $21(2013) 24$.

[16] C. Tsay, Y. Zha, C. B. Arnold. Opt. Express 18 (2010) 25.

[17] P. Ma, D. Y. Choi, Y. Yu, Z. Yang, K. Vu, T. Nguyen, A. Mitchell, B. Luther-Davies, S. Madden. Opt. Express. 23 (2015) 15.

[18] H. Lin, L. Li, Yi Z, S. Danto, J. D. Musgraves, K. Richardson, S. Kozacik, M. Murakowski, D. Prather, P. T. Lin, V. Singh, A. Agarwal, L. C. Kimerling, J. Hu. Opt. Lett. 38 (2013) 9.

[19] Anne, M. L., J. Keirsse, V. Nazabal, K. Hyodo, S. Inoue, C. Boussard-Pledel, H. Lhermite, J. Charrier, 
K. Yanakata, O. Loreal, J. Le Person, F. Colas, C. Compere, B. Bureau. Sensors. 9 (2009) 9.

[20] D. Zhang, L. Men, Q. Chen. Sensors. 11 (2011) 5360-5382.

[21] B. Pejcic, M. Myers, A. Ross. Sensors, 9 (2009) 6232-6253.

[22] World Health Organization. Guidelines for Drinking Water Quality. Available on line: http://www.who.int/water_sanitation_health/dwq/chemicals/toluene.pdf p.9 (accessed September 2016).

[23] ASHRAE Standard 62.1-2013. Ventilation for Acceptable Indoor Air Quality (2013).

[24] Carbon dioxide in indoor air. ANSES - French Agency for Food, Environmental and Occupational Health \& Safety (2013). Available on line: https://www.anses.fr/fr/system/files/AIR2012sa0093Ra.pdf p.1 (accessed April 2016).

[25] S. Prasad, L. Zhao, J. Gomes. Epidemiology. 22 (2011) S251.

[26] International Chemical Safety Cards (ICSC) of the National Institute for Occupational Safety and Health (NIOSH). Methane. ICSC \# 0291. CAS \#: 74-82-8 (2010). Available on line: http://www.cdc.gov/niosh/ipcsneng/neng0291.html (accessed December 2015)

[27] V. M. N. Passaro, C. de Tullio, B. Troia, M. La Notte, G. Giannoccaro, F. De Leonardis. Sensors 12 (2012) 15558-15598.

[28] R. Siebert, J. Müller. Sensors and Actuators A. 119 (2005) 138-149.

[29] J. Charrier, M. L. Brandily, H. Lhermite, K. Michel, B. Bureau, F. Verger, V. Nazabal. Sensors and Actuators B. 173, (2012) 468-476.

[30] B. Mizaikoff. Meas. Sci. Technol. 10 (1999) 1185-1194.

[31] P. Nemec, M. Olivier, E. Baudet, A. Kalendova, P. Benda, V. Nazabal. Materials Research Bulletin, 51 (2014) 176-179.

[32] P. E. Ciddor. Appl. Optics 35 (1996) 1566-1573.

[33] G. M. Hale, M. R. Querry. Appl. Opt. 12 (1973) 555-563.

[34] L. Jiang, S. Pau. Appl. Phys. Lett. 90 (2007) 111108. 
[35] M. Alrefae, E. Es-sebbar, A. Farooq. J. Mol. Spectrosc. 303 (2014) 8-14.

[36] M. M. Geenber. Environ. Res. 72 (1997) 1-7.

[37] M. Gerin, J. Siemiatycki, M. De’sy, D. Krewski. Am. J. Ind. Med. 341 (1998) 144-156.

[38] J. E. Bertie, R. N. Jones, Y. Apelblat, C. D. Keefe. Appl. Spectrosc. 281 (1994) 127-143.

[39] D. M. Wieliczka, S. Weng, M. R. Querry. Appl. Opt. 28 (1989) 1714-1719.

[40] R. Gobel, R.W. Seitz, S.A. Tomellini, R. Krska, R. Kellner. Vib. Spectrosc. 8 (1995) 141-149.

[41] E. Baudet, A. Gutierrez-Arroyo, P. Němec, L. Bodiou, J. Lemaitre, O. De Sagazan, H. Lhermitte, E. Rinnert, K.

Michel, B. Bureau, J. Charrier, and V. Nazabal, Opt. Mater. Express 6, (2016) 2616-2627.

[42] V. Acha, M. Meurens, H. Naveau, S. N. Agathos. Biotechnol. Bioeng. 685 (2000) 473-487. 